\title{
Design and Voluntary Motion Intention Estimation of a Novel Wearable Full-Body Flexible Exoskeleton Robot
}

\author{
Chunjie Chen, ${ }^{1,2,3}$ Xinyu Wu, ${ }^{1,2,4}$ Du-xin Liu, ${ }^{1,2,3}$ Wei Feng, ${ }^{1,2}$ and Can Wang \\ ${ }^{1}$ Guangdong Provincial Key Laboratory of Robotics and Intelligent System, Shenzhen Institutes of Advanced Technology, \\ Chinese Academy of Sciences, Shenzhen 518055, China \\ ${ }^{2}$ Chinese Academy of Sciences (CAS) Key Laboratory of Human-Machine Intelligence-Synergy Systems, Shenzhen 518055, China \\ ${ }^{3}$ Shenzhen College of Advanced Technology, University of Chinese Academy of Sciences, Beijing, China \\ ${ }^{4}$ Department of Mechanical and Automation Engineering, The Chinese University of Hong Kong, Hong Kong
}

Correspondence should be addressed to Xinyu Wu; xy.wu@siat.ac.cn and Can Wang; can.wang@siat.ac.cn

Received 28 January 2017; Accepted 30 March 2017; Published 27 June 2017

Academic Editor: Qiang Liu

Copyright (C) 2017 Chunjie Chen et al. This is an open access article distributed under the Creative Commons Attribution License, which permits unrestricted use, distribution, and reproduction in any medium, provided the original work is properly cited.

The wearable full-body exoskeleton robot developed in this study is one application of mobile cyberphysical system (CPS), which is a complex mobile system integrating mechanics, electronics, computer science, and artificial intelligence. Steel wire was used as the flexible transmission medium and a group of special wire-locking structures was designed. Additionally, we designed passive joints for partial joints of the exoskeleton. Finally, we proposed a novel gait phase recognition method for full-body exoskeletons using only joint angular sensors, plantar pressure sensors, and inclination sensors. The method consists of four procedures. Firstly, we classified the three types of main motion patterns: normal walking on the ground, stair-climbing and stair-descending, and sit-to-stand movement. Secondly, we segregated the experimental data into one gait cycle. Thirdly, we divided one gait cycle into eight gait phases. Finally, we built a gait phase recognition model based on $k$-Nearest Neighbor perception and trained it with the phase-labeled gait data. The experimental result shows that the model has a $98.52 \%$ average correct rate of classification of the main motion patterns on the testing set and a $95.32 \%$ average correct rate of phase recognition on the testing set. So the exoskeleton robot can achieve human motion intention in real time and coordinate its movement with the wearer.

\section{Introduction}

The number of China's elderly and disabled people had reached 260 million at the end of 2014, China's rapid aging has caused widespread concern, and it is difficult for China to afford the issue of a rapidly aging population [1]. Additionally, the number of hemiplegic and paraplegic patients and people with walking difficulties increased year by year. Most of these people can only use a wheelchair to achieve self-care. Walking and standing are their greatest desire, the physical function of the elderly declines, and their daily activities are limited with the increase of age. It brings great pressure and burden to the families and society to take care of the elderly, which has become a major social problem. Thus, it is necessary to develop a power exoskeleton device to assist the elderly in their needs. The exoskeleton robot is a kind of wearable human-machine integration device, which combines the science of robotics and rehabilitation engineering. The application of an exoskeleton robot can help the patients who have lost their walking ability or have walking difficulties stand and walk normally again.

The concept of mobile cyberphysical system (CPS) has emerged as a promising tool where the operations of the physical and engineered systems are monitored, controlled, coordinated, and integrated by means of a computing and communication core [2]. The exoskeleton robot must determine the wearer's moving intention accurately and quickly due to its close link with the wearer. Recent advances in microelectromechanical systems (MEMS) have led to the rapid development of microsensors [3, 4]. Correlations among the data representing an event are usually reflected in multiple measurements at different locations $[5,6]$. The 
acquisition of electroencephalogram, electromyography, and other biological signals have become a hot research topic in the field of human-machine interaction owing to their quick response $[6,7]$. However, the measurement of biological signals involves poor robustness because of their low frequency, weak amplitude, and low signal-to-noise ratio [8]. On the other hand, the traditional force feedback and position tracking control strategies are also widely used for exoskeleton control, which is based on physical signals; a wearer with walking difficulties has difficulty in achieving free walking by simply relying on physical signals because of physical signals' obvious signal delay and too many sensors. As research based on biological signals, force, position, and other physical signals has not yet achieved breakthroughs in the motion intention estimation for exoskeleton robots, researchers have been searching for new methods to assess the wearer's moving intentions accurately and quickly.

The Cybernetics Laboratory of Tsukuba University developed the HAL series of wearable power-assist robot systems to help the elderly or the disabled to achieve normal walking in 2002. This exoskeleton robot can help disabled people stand up or reduce the labor intensity of workers. The control strategy of the HAL series includes two kinds of control modes: one is based on EMG and the other one involves gait prelearning and gait pattern generation. Researchers who developed HAL series established a joint torque control method based on EMG signals to achieve control and maintain coordination with the wearer's lower limb movement [9].

In the field of exoskeleton robots research, one walking gait cycle is usually divided into multiple phases. In [10], the walking gait cycle was divided into the stance phase and the swing phase. Murray et al. [11] proposed an assistive approach without dictating the spatiotemporal nature of joint movement for the lower limb exoskeleton; a finite-state machine consisting of six gait phase states was used to govern the exoskeleton controller. Kazerooni et al. [12] adopted a hybrid control strategy for different gait phases to control the Berkeley Lower Extremity Exoskeleton. Liu et al. [13] proposed an approach of gait phase recognition for a lower limb exoskeleton with only joint angular sensors. Oh et al. [14] considered that a single control method is not the best option for all motion phases during the gait cycle. It is difficult to use a fixed model to describe the process of walking and obtain a fixed output setting due to individual differences in human walking and the changes in road conditions. Normal walking is the result of a coordinated body movement that allows the body to move in the most efficient way [15]. In this study, we developed a set of novel wearable fullbody flexible exoskeleton robots and presented a method of voluntary motion intention estimation, which can help wearable exoskeleton robot walk comfortably.

\section{Materials and Methods}

2.1. Design of a Novel Wearable Full-Body Flexible Exoskeleton Robot. The human body has three basic planes: the sagittal plane, the coronal plane, and the horizontal plane, and these three surfaces are perpendicular to each other at the center
TABLE 1: Adjustment range of the connecting rod of the exoskeleton robot.

\begin{tabular}{lc}
\hline Adjustable Component & Range \\
\hline Lower leg & $340 \mathrm{~mm}-410 \mathrm{~mm}$ \\
Thigh & $315 \mathrm{~mm}-385 \mathrm{~mm}$ \\
Upper arm & $318 \mathrm{~mm}-379 \mathrm{~mm}$ \\
Forearm & $218 \mathrm{~mm}-261 \mathrm{~mm}$ \\
\hline
\end{tabular}

of the body [16]. The exoskeleton robot is positioned on the human body and the wearer coordinates his movement with the robot; the design of the robot's dimensions must be in accordance with the specifications of the human body. The length of the connecting rod of a full-body exoskeleton robot should be adjustable to a certain range for better compatibility. The size design of the exoskeleton in this paper refers to the GB10000-88 (Chinese adult body size) standard. Although the size of various parts of the body and their proportions are basically fixed, there are differences in proportions of the body between men and women; the lower limb size of men is generally larger than that of women [17]. The height range of a subject was limited from 1550 to $1850 \mathrm{~mm}$, and then the regulating length range of the connecting rod can be calculated as Table 1 .

The exoskeleton robot must be a mechanical shadow of the wearer and it must be able to mimic each of his actions in real time, and even a millisecond's hesitation can create a burden that makes the wearer feel as impeded as walking in water. Therefore, its sensors must be able to quickly read every minor action and its microprocessor must be sufficiently powerful to convert these data into instructions to the robot in real time. The speed of traditional hydraulic transmission is too slow which increases the weight of robot; additionally, if a motor drive was mounted directly on the joint that will require a larger body structure. Therefore, in this study, we positioned all the drive units in the backpack to obtain a more delicate exoskeleton robot system.

Figure 1 shows the overall structure of the exoskeleton robot system. The system uses a distributed control architecture, which is composed of the main control computer, data acquisition board, motor drive board and various sensors, and so forth.

2.1.1. Steel Wire Transmission. The steel wire and tube are forced along the tangent direction of the wire line when the motor drives the wire. The tube is rigid and incompressible in the longitudinal direction although the wire and tube appear to be soft, the interior of the tube is made of steel, and the wall is rather thick. The steel wire and the tube can be bent only to a limited degree according to different types, while the deformation of the steel wire is negligible compared to the moving distance of the motor output. As a result, the motor torque can be transferred to the terminal almost without loss [18].

The steel wire in one loop is divided into three parts: the upper end of the wire between the upper part of tube and the lower tube, the tube, and the part between the tube and the lower end of the lower part of the wire, their length is $L_{1}$, 


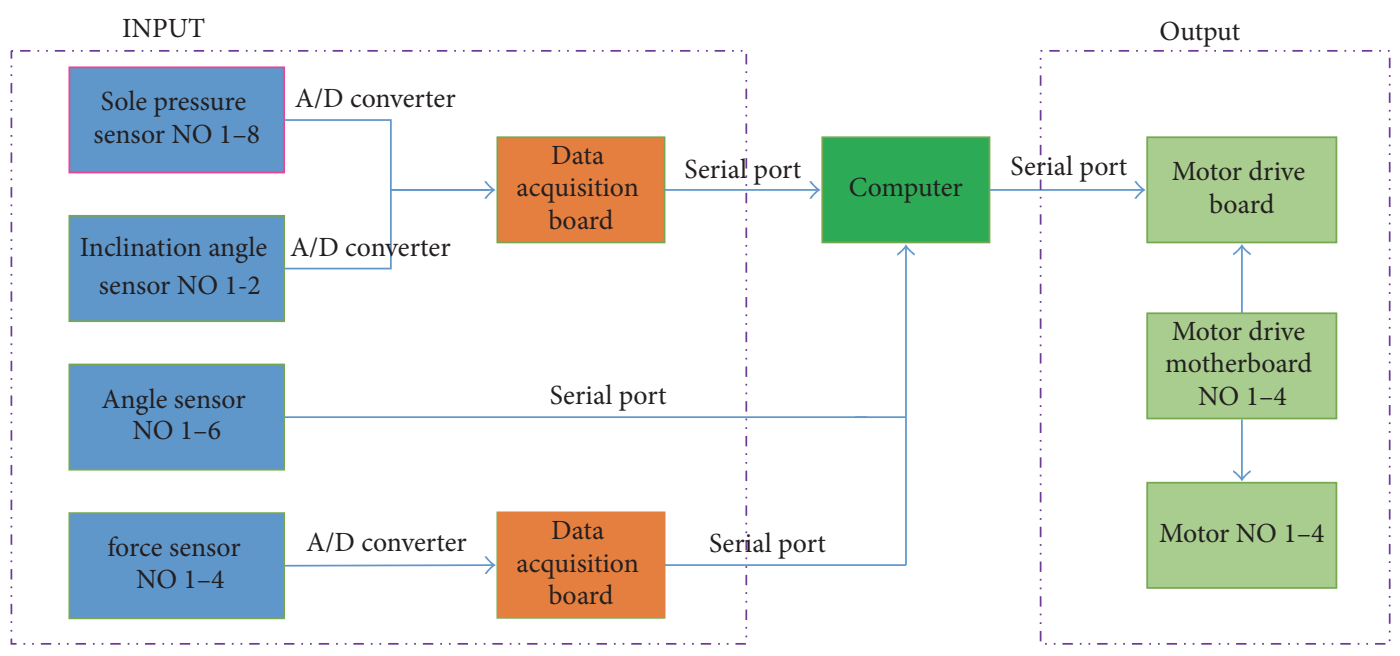

FIGURE 1: Exoskeleton robot system structure.

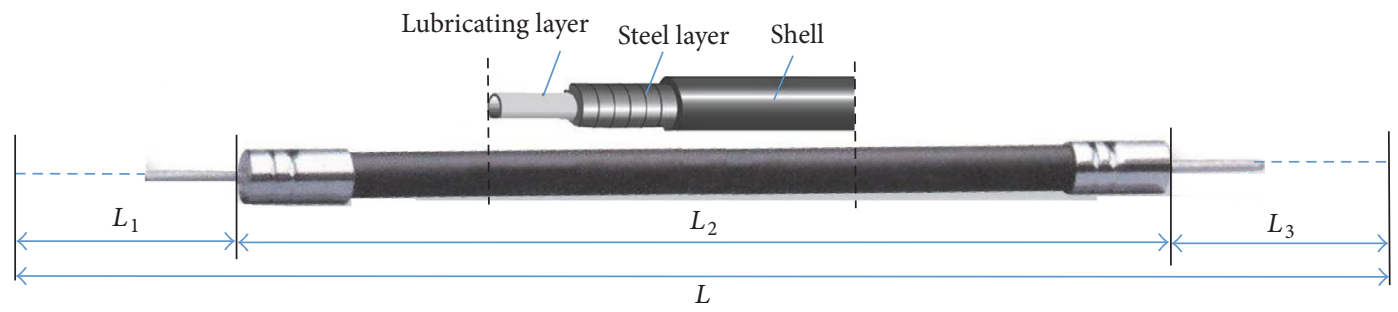

FIGURE 2: Steel wire in one loop.

$L_{2}$, and $L_{3}$, respectively, and the total length of steel wire is $L$ which is shown in Figure 2.

$$
L=L_{1}+L_{2}+L_{3} \text {. }
$$

$L_{1}$ will increase when the motor rotates, $L_{2}$ does not change because it is actually the length of the tube, and $L$ does not change because it is the length of the steel wire; consequently, $L_{3}$ has to decrease. Thus, a wire drive circuit is formed.

The advantage of this transmission approach described in this paper is combined with hydraulic and pneumatic transmission, gears drive, and any other traditional transmission, which can realize flexible transmission with a rather simple structure as shown in Figure 3. Additionally, it can avoid the noise caused by hydraulic pumps or air pumps. Therefore, it constitutes a major innovation for the structural design of the exoskeleton robot, which can easily transfer the torque to any joint of the robot without interfering with the other joints.

Most steel wire transmission systems achieve the torque transmission through a pulley or pulley group. Furthermore, most of steel wire transmission systems involve only oneway transmission, which require a spring or other devices to assist in the return time of a loop, and they also need large pretightening force of the steel wire to prevent slipping. In this study, we designed a group of special wire-locking pulley structures as shown in Figure 4 that resulted in a wire without

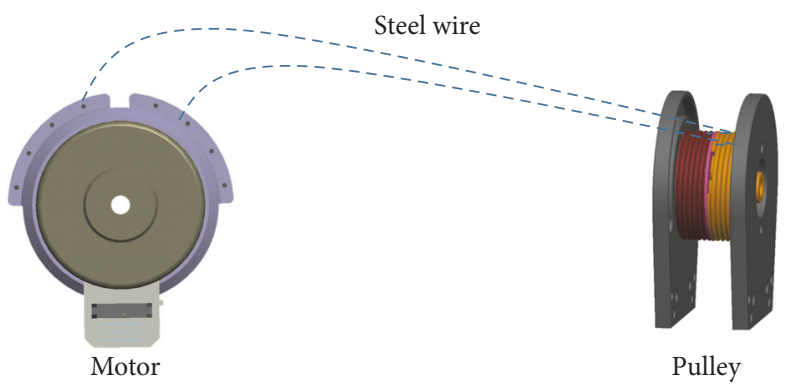

Figure 3: Steel wire transmissions.

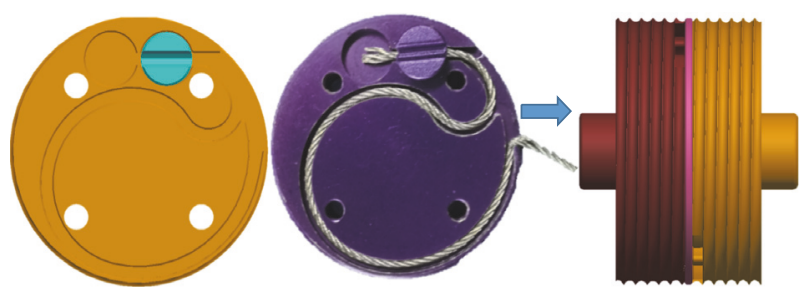

FIGURE 4: Wire-locking pulley.

slippage; thus, transmission could be fully implemented as needed. 


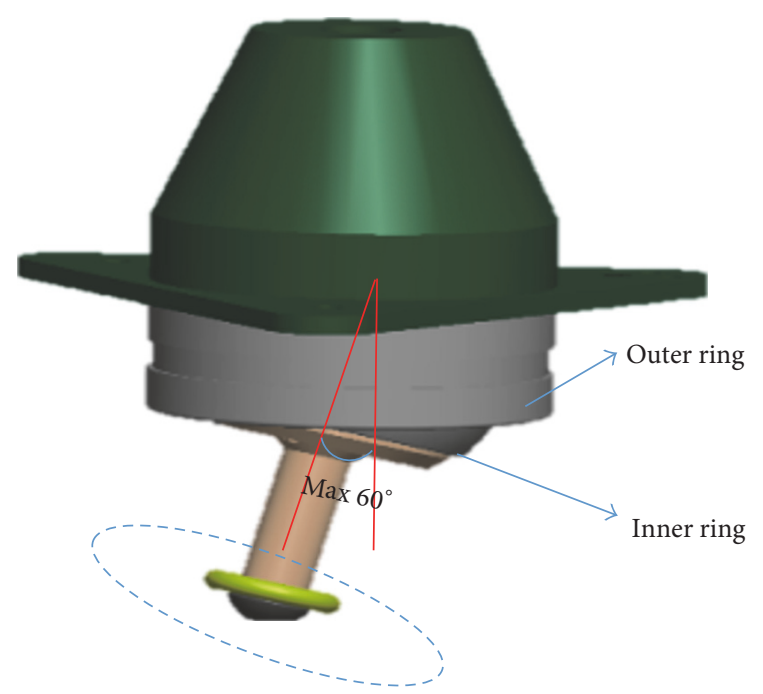

FIGURE 5: Passive spherical joint.

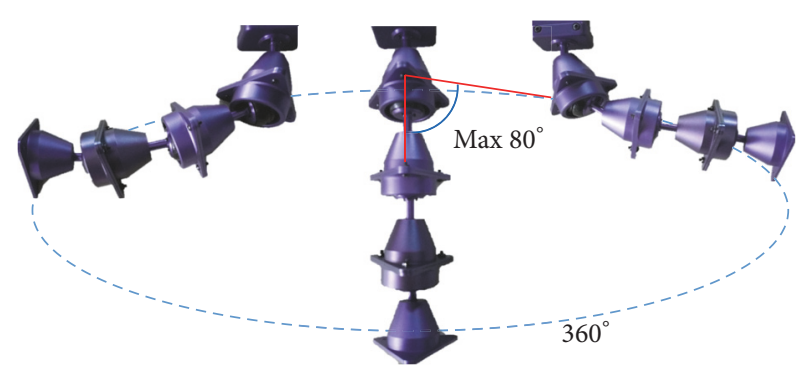

FIgURE 6: Passive spherical joint group.

2.1.2. Passive Spherical Joint. This joint is composed of a special sliding bearing structure containing an inner ring with an outer spherical surface and an outer ring with an inner sphere. The joint which is shown in Figure 5 has two bonded spherical surfaces that can withstand large loads and it is usually used for the low speed of the swing movement. It can also be tilted in a certain angle because of the sliding surface of the spherical shape, which can operate normally even when the support shaft and the shaft shell hole are not concentric. Several joints can be connected to a group as needed, which is shown in Figure 6.

This type of joint is very suitable for the flexible robot developed in this study, which can be worn without being bound by the mechanical structure of exoskeleton robot, and it greatly improves comfort to the wearer.

2.1.3. System Overview. The wearable exoskeleton robot developed in this study is a complex intelligent system integrating mechanics, electronics, computer science, and artificial intelligence, which is necessary to capture the movement state and motion intention of the wearer through signals from the sensor system. The sensor system mainly includes angle sensors, inclination sensors, plantar pressure sensors, and pressure sensors between the human limbs and the

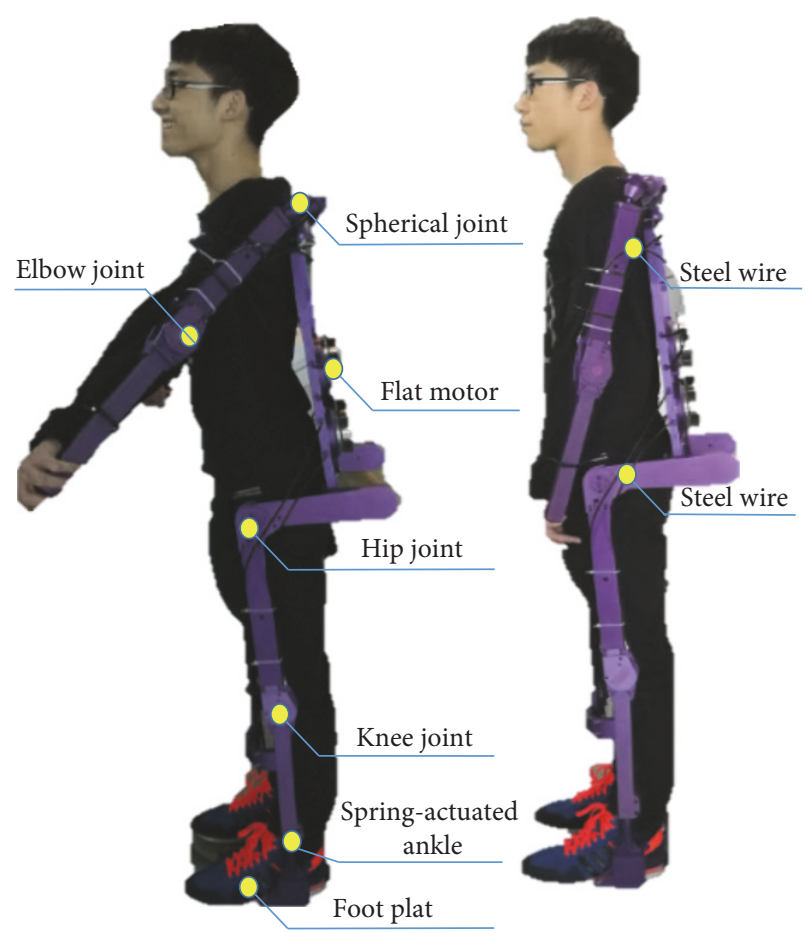

FIGURE 7: Full-body flexible exoskeleton robot.

exoskeleton robot as shown in Figure 7. The gait acquisition system was established in advance, and the sensors were installed at each joint of the gait acquisition system, the gait acquisition system could adapt to wearers of different heights, and the following are the main tasks of the four kinds of sensors:

(1) The inclination sensors help detect body posture and prevent falling.

(2) The plantar pressure sensors are used to determine the motion state.

(3) The joint angle sensors are used to determine the joint angle of the robot.

(4) The pressure sensor is used to collect the real-time interaction data between the human limb and the exoskeleton robot.

Energy consumption of the knee joint is the most of all the joints according to the data curve of the joint movement angle and output torque [19]. The exoskeleton robot is difficult to be designed with the same freedom as with human shoulder using only a motor drive because of complex human body structure, whereas the elbow joint movement is simple. The exoskeleton robot developed in this study was designed to only add active driving in the knee and elbow joints, and we used flexible passive degrees of freedom for all the other joints.

2.2. Sensor Data Acquisition System and Experiment. The lower limb of human is generally believed to have seven degrees of freedom [20]: hip adduction, abduction, external 


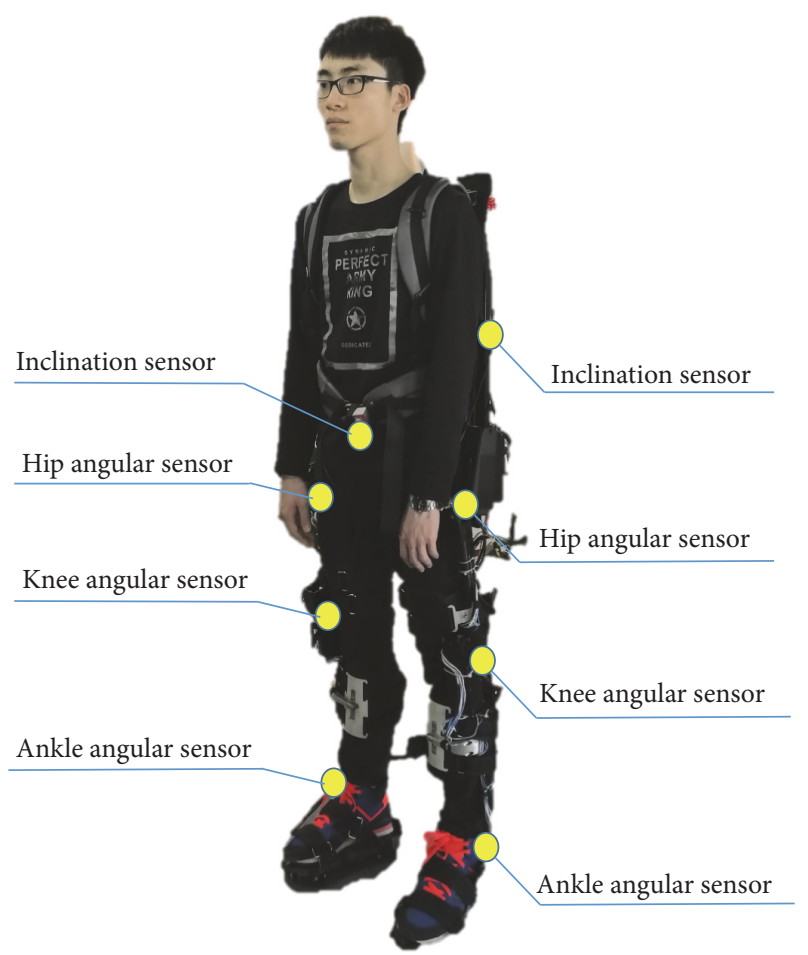

FIGURE 8: Sensor data acquisition system.
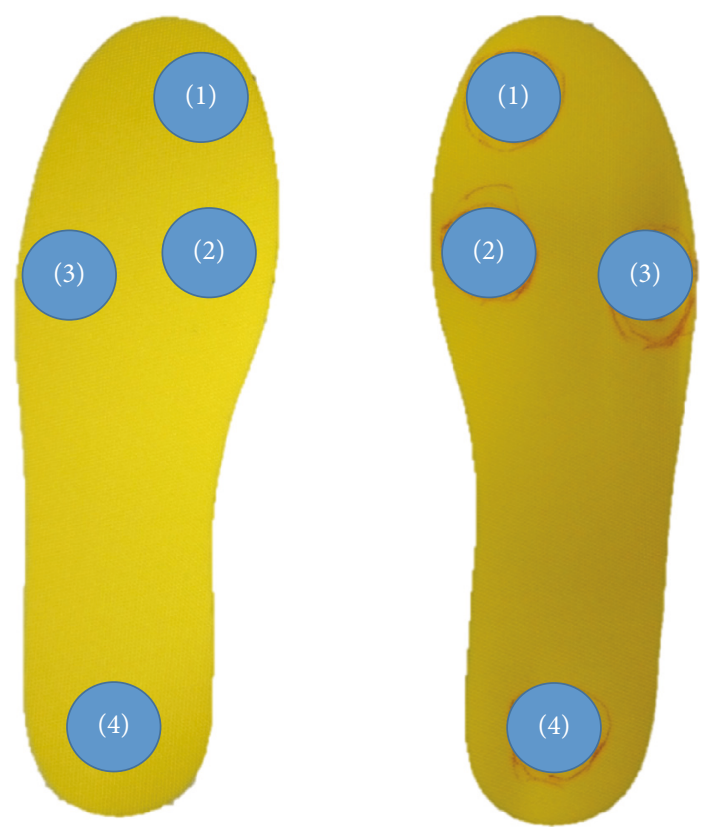

Figure 9: Plantar pressure sensor distribution.

rotation, internal rotation, ankle valgus and virus, external rotation, and internal rotation motion. The angles of the hip, knee, and ankle changed regularly during the walking. We only collected data from the angle of the hip, knee, and ankle in the sagittal plane as shown in Figure 8 to facilitate the analysis of the gait. The distribution of plantar pressure sensors is shown in Figure 9. In walking, the upper part of the
TABLE 2: Exoskeleton sensor data.

\begin{tabular}{lc}
\hline Type & Number \\
\hline Plantar pressure & 8 \\
Joint angle & 6 \\
Inclination angle & 4 \\
\hline
\end{tabular}

body exhibits a slight swing with the swing of the feet [21]. We installed sensors to acquire upper body swinging data to more accurately reproduce the process of walking. We collected and analyzed the changes of the hip, knee, ankle angle, and body posture that occur in the process of walking, stairclimbing, and sit-to-stand (STS) movement to understand the real human gait; Table 2 shows the number of three types of sensors.

Subjects. All the recruited subjects before the experiment were informed about the experimental nature, procedure, effect, and the potential risk. They were very willing to participate in experiment and sign the informed consent forms. Meanwhile, this experiment has been approved by Shenzhen Institutes of Advanced Technology Ethics Committee.

We selected 10 young men with normal lower limb function to perform walking on the ground, treadmill walking, stair-climbing and stair-descending, and STS tests using our sensor data acquisition system. The mean age of the group was 25.3 years, with an average height of $173.3 \mathrm{~cm}$ and an average weight of $67.7 \mathrm{~kg}$. Before the experiment, the participants' thigh and leg length and waist thickness were measured, and the various links of the collector were adjusted to the appropriate size to conduct the experiment.

Gait analysis is a method to study walking patterns that aims to reveal the key links and factors affecting gait by means of mechanics and kinematics. This technique is also helpful to guide the robot in the later control stage. Compared with other biometrics, such as fingerprint, iris, and face recognition, gait represents a type of external dynamic performance that is closely related to spatial and temporal information. Gait recognition must begin with the lower limb of the human body and the gait signal is divided periodically. It must accurately and quickly determine the wearer's movement intentions and make decisions because the robot has a close relationship with the wearer. People always maintain the center of gravity while walking [22]. In the initial stage of the gait, the wearer is not yet skilled at controlling the robot. The motion planning algorithm requires more intervention and learning to adapt to the gait. The algorithm will gradually stabilize and finally achieves a regular gait when a stable complete gait is achieved.

\subsubsection{Walking at Different Speeds on the Ground and a Tread-} mill. The walking gait of a human has the characteristics of periodicity, left-right symmetry, and coordination, and the walking motion is mainly performed in the sagittal plane [23]. The time of the process that the same heel touches the ground again is called a complete gait cycle during walking. Both sides have their own gait cycle as the left and right legs 


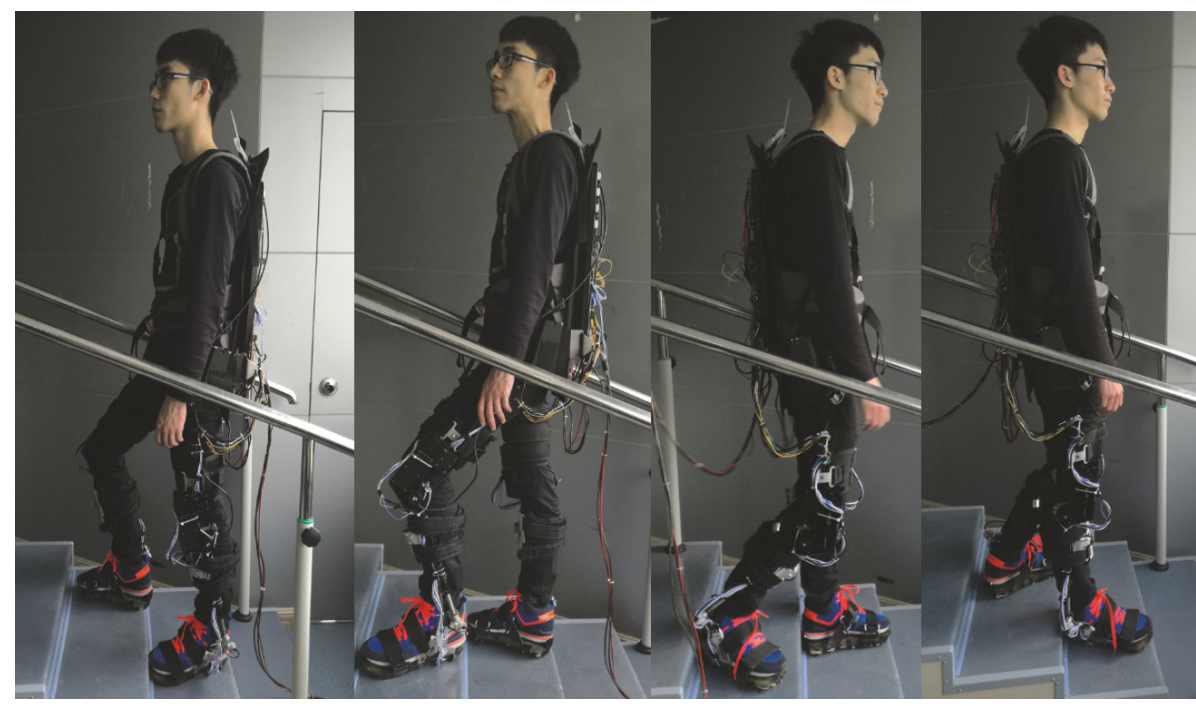

FIGURE 10: Stair-climbing and stair-descending.
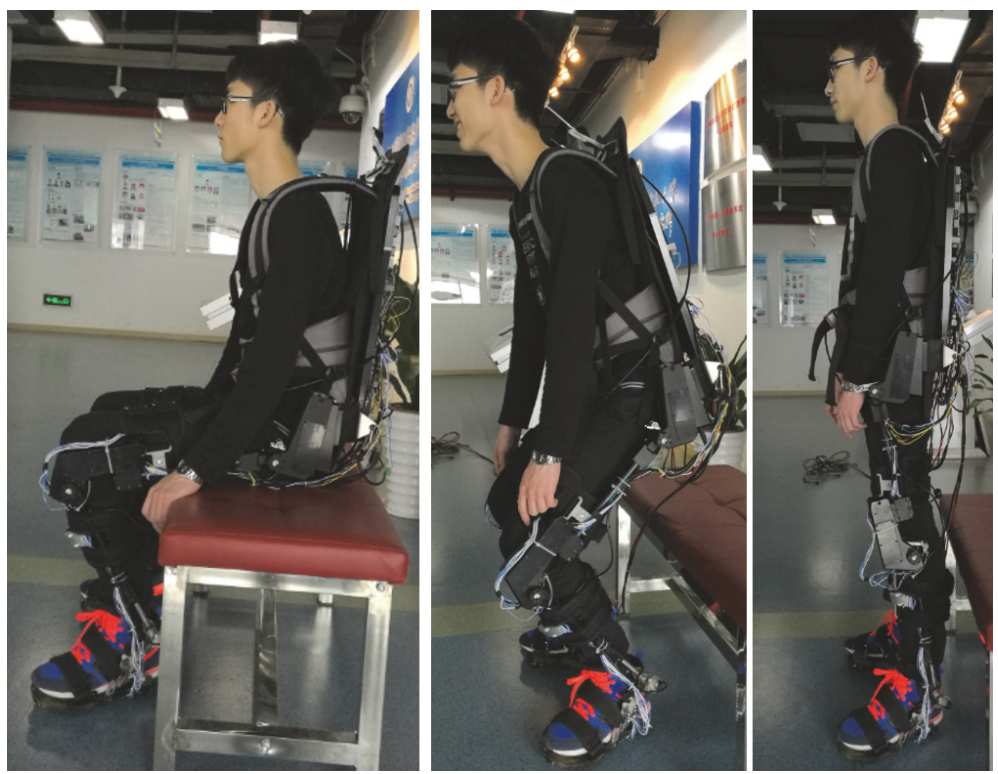

FIGURE 11: STS.

alternate. The right lower limb is considered as an example because of the symmetry of human gait; the same person's left and right leg gait are usually only a phase difference.

2.2.2. Stair-Climbing and Stair-Descending. The cyclic pattern of the lower limbs during the task of stair-climbing and stairdescending as shown in Figure 10 is very similar to the pattern observed during walking on the ground [24].

For both tasks, periods of support (stance) and nonsupport (swing) can be defined. Joseph and Watson have cited similar figures (i.e., $60 \%$ stance, $40 \%$ swing) for a stairdescending task. In contrast, for the lower limbs of subjects in stairs-climbing task it was observed that $66 \%$ time of the gait cycle is stance phase and the remaining $33 \%$ time is the swing phase [25].
2.2.3. STS. STS movement is also divided into phases as shown in Figure 11 for better understanding, the most commonly used classification for which contains two phases [26], one is the preparation phase, which includes the flexion process of the upper part of the body and the process of the body begins the seat-off motion, the other one is the rising phase, which contains the process from the seat-off action (maximum anterior flexion of the trunk) to the standing posture, and finally people maintain their body in a quasistationary position.

2.3. Voluntary Motion Intention Estimation. The phases of the gait data are firstly classified offline before the motion intention estimation. 


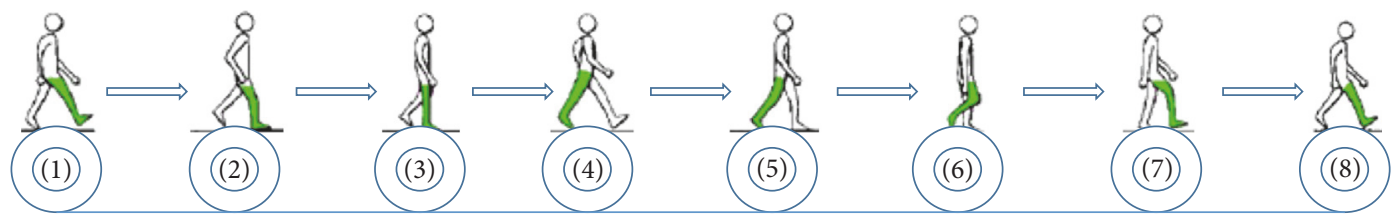

FIGURE 12: Human walking gait cycle.

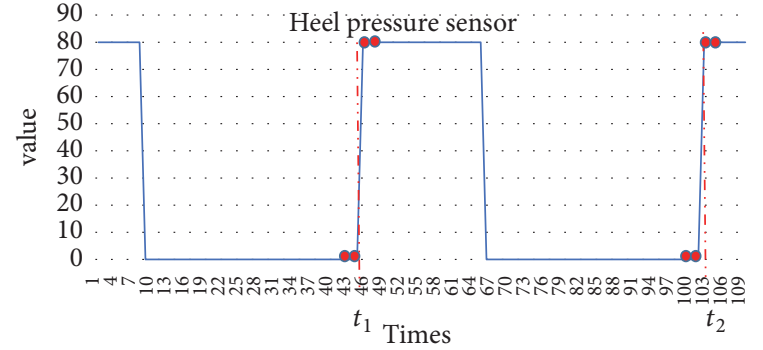

FIGURE 13: Gait cycle cutting.

2.3.1. Gait Cycle Division. The lower limbs repetitively deviate from the standard standing position in walking [27]. Gait of walking is a cyclic phenomenon that can be divided into phases from the initial contact with the floor to the final contact with the floor in the swing phase; it begins when the tibia is vertical to the floor and ends when the foot strikes the floor again.

In this study, one gait cycle was divided into eight phases as shown in Figure 12; the proportion of each phase in one gait cycle is as follows:

(1) Initial contact phase ( $0 \%)$.

(2) Loading response phase (0-10\%).

(3) Middle stance phase (10-30\%).

(4) Terminal stance phase (30-50\%).

(5) Preswing phase (50-60\%).

(6) Initial swing phase (60-73\%).

(7) Middle swing phase (73-87\%).

(8) Terminal swing phase (87-100\%).

One gait cycle is defined by the time that the right heel hits the ground two times. The moment of mutation is used as the dividing point according to the data of the plantar pressure sensors. The gait sequence can be automatically divided into several gait cycles by using this method. We defined the moment when two consecutive point values of the heel pressure sensors are greater than the setting threshold as the dividing point which is shown in Figure 13.

$$
\begin{aligned}
T_{\text {cycle }} & =t_{i+1}-t_{i}, \quad i=1,2, \ldots, N, \\
\Delta t_{i} & =\left(\Delta t_{1}, \Delta t_{2}, \Delta t_{3}, \ldots, \Delta t_{n}\right) .
\end{aligned}
$$

We can divide the gait cycle as required by determining two such successive points. From the 10 recruited subjects that performed the walking test with the sensor data acquisition system, 4500 gait cycles were obtained. Each captured gait cycle dataset can be expressed in the form of a matrix.

$$
\left[\begin{array}{ccc}
s 1 & \cdots & s 18 \\
\vdots & \ddots & \vdots \\
s 1 j & \cdots & s 18 j
\end{array}\right], \quad j=1,2, \ldots, N
$$

where $j$ is the number of data points of each captured gait cycle, which depends on the walking speed and sampling frequency, and $s 1$ to $s 18$ are all the data of the sensors installed in the sensor data acquisition system.

2.3.2. Gait Phase Labels on Gait Data. We verified the classification method after dividing the gait cycle. Each of the obtained 4500 gait cycles was divided into eight phases by the proportion of time in one gait cycle.

One gait cycle was taken for analysis; a gait cycle determined with the above method begins approximately at the moment that one foot leaves the ground and ends with the ipsilateral foot leaving the ground. The gait phase classification resulted in the values as shown in Figure 14. The cyclic pattern of the lower limbs during the task of stairclimbing and stair-descending is very similar to the pattern of walking on the ground [28]. Therefore, we used the same proportion of time in a cycle as normal walking in this study. The STS movement was also divided into two phases for better understanding [28]. The first phase is the flexion phase, which occurred during the first 35\% of the STS cycle. The second phase is the extension phase; a gait cycle is also generally divided into two main phases: the stance phase (60\%) and the swing phase (40\%) [29]. The opposite process applies and the phases are easy to divide during the process of standing up and sitting down.

2.3.3. Gait Phase Recognition. In the actual movement process, we can use the following methods to obtain the realtime behavior of the robot once the instantaneous sensor data information is obtained. In this study, all the actions were divided into the three categories of normal walking on the ground, stair-climbing and stair-descending, and STS. Firstly, we used the following method to distinguish these three categories. In each category, the phase in the gait cycle needs to be found out in real time; walking on the ground was taken as an example, and then Figure 15 shows how we further identified the specific gait phases, the eight different color blocks represent the eight phases in a gait cycle, the 


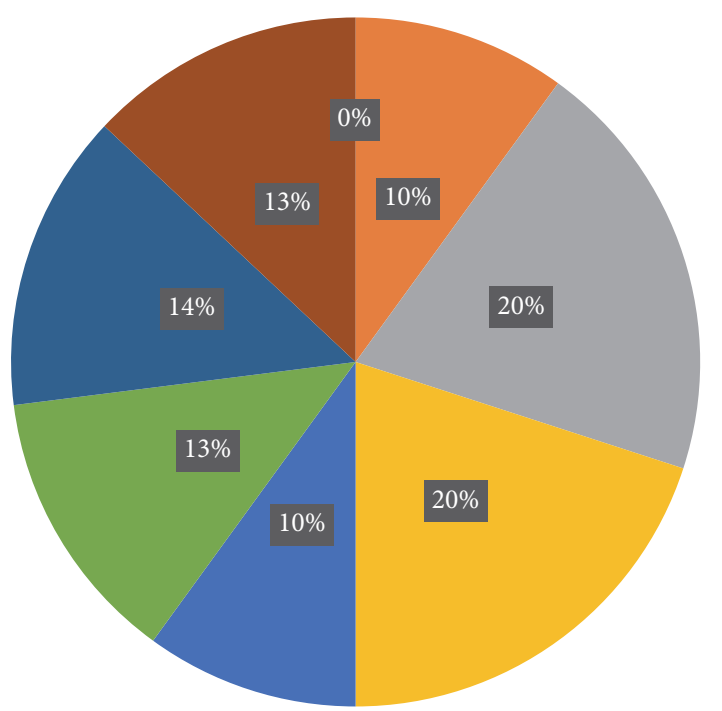

(1) Initial contact phase

(2) Loading response phase

(3) Middle stance phase

(4) Terminal stance phase

(5) Preswing phase

(6) Initial wing phase

(7) Middle swing phase

(8) Terminal swing phase

FIgURE 14: Gait phases in one cycle.

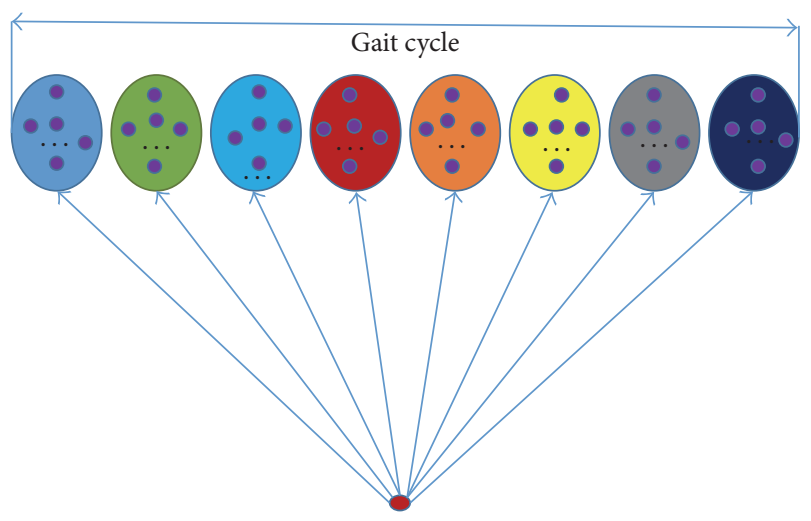

FIGURE 15: Gait phase recognition.

blue dot inside the blocks represents the specific data points in each phase, the nearest blue point to the target point was found by calculating, and the same method is also applied to other categories.

Distinguishing of Walking on the Ground, Stair-Climbing, and Stair-Descending. A novel method was developed to distinguish these two categories as shown in Figure 16 based on the gait cycle classification data described above and then a $k$-Nearest Neighbor classifier was created for data training.

$$
\begin{aligned}
\theta i & =\boldsymbol{\theta}_{\mathrm{LK} i}-\boldsymbol{\theta}_{\mathrm{RK} i} \quad i=1,2, \ldots, N, \\
\Delta \boldsymbol{\theta} \mathbf{i} & =(\Delta \boldsymbol{\theta} 1, \Delta \boldsymbol{\theta} 2, \Delta \boldsymbol{\theta} 3, \ldots, \Delta \boldsymbol{\theta} n),
\end{aligned}
$$

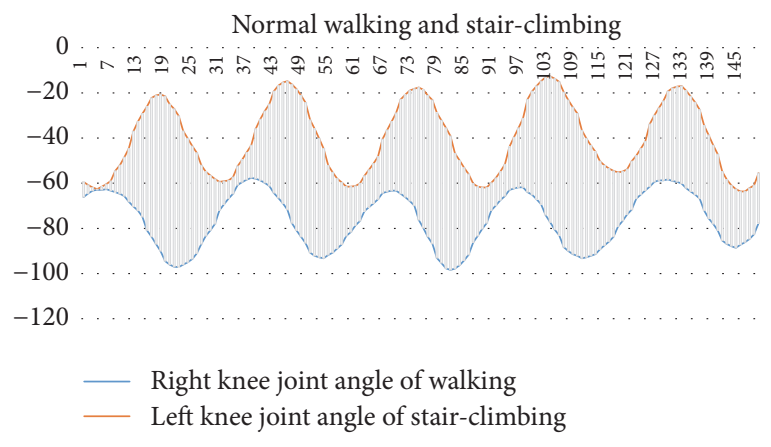

FIGURE 16: Distinguishing of normal walking and stair-climbing.

where $\boldsymbol{\theta}_{\mathrm{LK}}$ denotes the left knee joint angle for stair-climbing and $\boldsymbol{\theta}_{\mathrm{RK}}$ indicates the right knee joint angle for normal walking.

STS. Standing from a seated position is an activity that we perform many times every day. STS is a rather action different from normal walking and stair-climbing and stairdescending; the angular values that we recorded reflected the relationships of the lower limb joints. This difference was confirmed by a reversal of the rapid increase of angle in knee extension. The angle of the hip and ankle joints also increased. The left and right leg have the same phase during the STS action; this is the greatest difference between STS and other actions, in which the two legs have the same phases. Thus, we can easily distinguish STS categories.

$$
\begin{aligned}
& \boldsymbol{\theta}_{\mathrm{LA}}=\boldsymbol{\theta}_{\mathrm{RA}}, \\
& \boldsymbol{\theta}_{\mathrm{LK}}=\boldsymbol{\theta}_{\mathrm{RK}}, \\
& \boldsymbol{\theta}_{\mathrm{LH}}=\boldsymbol{\theta}_{\mathrm{RH}},
\end{aligned}
$$

where $\boldsymbol{\theta}_{\mathrm{LA}}$ denotes the left ankle joint angle, $\boldsymbol{\theta}_{\mathrm{RA}}$ is the right ankle joint angle, $\boldsymbol{\theta}_{\mathrm{LK}}$ is the left knee joint angle, $\boldsymbol{\theta}_{\mathrm{RA}}$ indicates the right knee joint angle, $\boldsymbol{\theta}_{\mathrm{LH}}$ is the left hip joint angle, and $\boldsymbol{\theta}_{\mathrm{RH}}$ denotes the right hip joint angle.

\section{Results and Discussion}

We observed that angle changes of the hip joint, knee joint, and ankle joint had obvious regularity in the process of walking through the analysis of the gait data. Furthermore, we verified that the walking gait described above was periodic and left-right symmetrical. In addition, we determined that there is a slight swing around the waist; the body naturally inclines to the side of the supporting leg to focus on the foot when people walk, which is to maintain the balance of the body and avoid falling.

We firstly divided them into normal walking, stairclimbing and stair-descending, and STS after obtaining the instantaneous 18-dimensional sensor data and then inserted them into the $K \mathrm{NN}$ model, which was built in a Python environment. We finally got a recognition rate of $98.21 \%$, which is nearly with no intersection of other phases, except the very standing stationary points that have the same phase. 


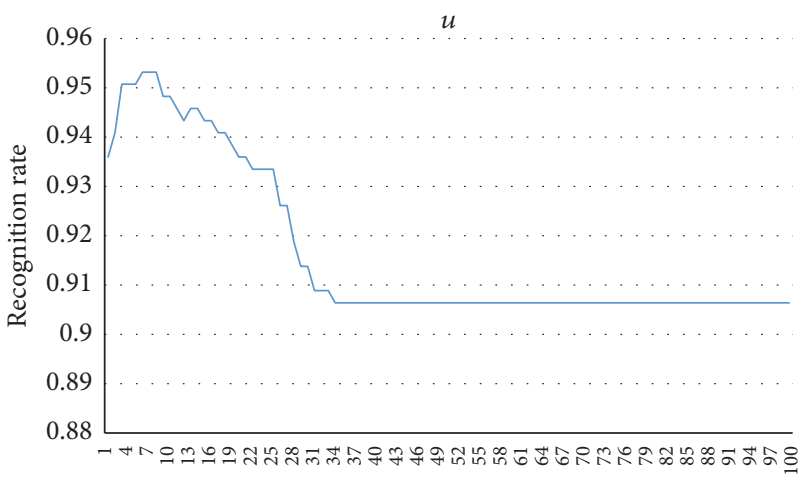

FIgURE 17: The best coefficient $u$.

Secondly, normal walking is taken as an example; we used the six angle sensors data to calculate the nearest point to the instantaneous point and then obtained the corresponding gait phase and the most probable point.

$$
\text { dist_angle }=\sqrt[2]{\sum_{1}^{6}\left(\text { test }_{i}-\text { target }_{i}\right)^{2}}, \quad i=1,2,3,4,5,6,
$$

where test ${ }_{i}$ denotes the data from the six ankle joint angles after training, target ${ }_{i}$ represents the instantaneous ankle joint angle data, and dist_angle indicates the distance between the target point and instantaneous point in the gait cycle.

$$
\begin{array}{r}
\text { dist_pressure }=\sqrt[2]{\sum_{1}^{8}\left(\text { test }_{j}-\operatorname{target}_{j}\right)^{2}}, \\
\quad j=1,2,3,4,5,6,7,8,
\end{array}
$$

where test ${ }_{j}$ represents the data from the eight plantar pressure sensors after training and target ${ }_{j}$ indicates the instantaneous plantar pressure sensor data.

In an actual gait, the plantar pressure sensors and the angle sensors data are not uniform, and the weight of the impact on the calculation results is unknown. Therefore, we must define a new coefficient $u$ :

$$
\text { dist }=\text { dist_angle }+ \text { dist_pressure } * u \text {, }
$$

where dist denotes the distance between the target point and instantaneous point and $u$ is the weight coefficient.

We inserted the target data into the training model and determined the best coefficient $u$ (from 1 to 100).

We calculated that the best recognition rate was $95.32 \%$ when $u=7,8,9$ and that the recognition rate no longer increases when $u \geq 35$; they are shown in Figure 17, so we did not choose the number larger than 100 to try. Finally, we selected $u=8$ and input four normal walking gait cycles into the model to identify the corresponding phase and pose, which provided the following result.

From Figure 18, we can easily see that the length of each gait cycle may be different according to the walking speed. We can acquire approximately 55 to 58 data points in a gait cycle while walking at a normal pace. The abscissa shows each point in the gait cycle; the ordinate is the every phase of the gait cycle. The blue curve shows the actual gait phases data, and the red one is predicted value through the gait phase recognition method.

In Figure 18(a) the middle stance phase exhibits recognition errors, while all the other phases are predicted accurately. In Figure 18(b), only the loading response phase and the preswing phase have small recognition errors and Figure 18(c) shows four gait phase recognition errors. However, each gait phase had only a few gait data points due to the sampling rate. Therefore, erroneously identified data points affected severely the correct recognition rate. In Figure 18(d), only one phase has a few recognition errors. From Figure 18, the correct rate of phase (CRP) of the four cycles was above $90 \%$ and the CRP of the entire gait set was $95.32 \%$. Further analysis determined that, in each of the phases that exhibited identification errors, only one gait data point was identified incorrectly. Therefore, the gait phase recognition model described in this paper was able to predict the gait phase labels accurately.

\section{Conclusions}

In this study, we designed a novel wearable full-body flexible exoskeleton robot as one application of mobile cyberphysical system (CPS). We used a signal acquisition system to collect data for normal walking on the ground, treadmill walking in a setting speed, stair-climbing and stair-descending, and STS. We employed a novel simple method to distinguish all kinds of action mentioned above according to the posture characteristics of angle and pressure. To date, many approaches have been developed to identify the gait phase. Here, we propose a novel gait phase recognition method using lower limb joint angle, plantar pressure, and inclination sensors. According to the characteristics of the gait data, we defined eight gait phases to extract the gait phase features. The deviation distances were calculated and classified by fixed proportion of time in one gait cycle. Then, the gait phase labels of the gait set were obtained. Through offline gait data classification, one gait cycle was divided into eight phases. We built a gait phase recognition model using the gait phase-labeled data. By training the model with a 14-dimensional input vector, we can recognize the gait phase in real time through the lower limb joint angle and plantar pressure sensor data. For the tested set, the model had a CRP of $95.32 \%$; the experimental results demonstrate the effectiveness of the gait phase recognition technique. This novel method can accurately and quickly assess the wearer's moving intentions with a rather simple sensor system. In future work, we intend to improve the existing control strategy of the full-body exoskeleton with the novel gait phase recognition method and develop a gait evaluation method for the exoskeleton robot. The same method can be applied to the upper-limb movement of the robot. Thus, the flexible full-body exoskeleton robot system will be realized in the near future. In the future, we can also use a mobile phone to receive the real-time sensor data of exoskeleton robot and choose the best control mode and walking route for the wearers. 


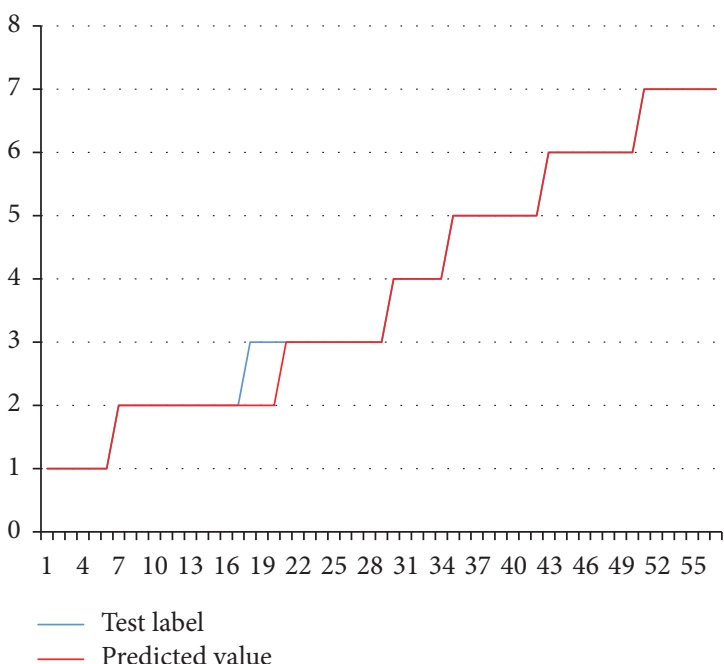

(a)

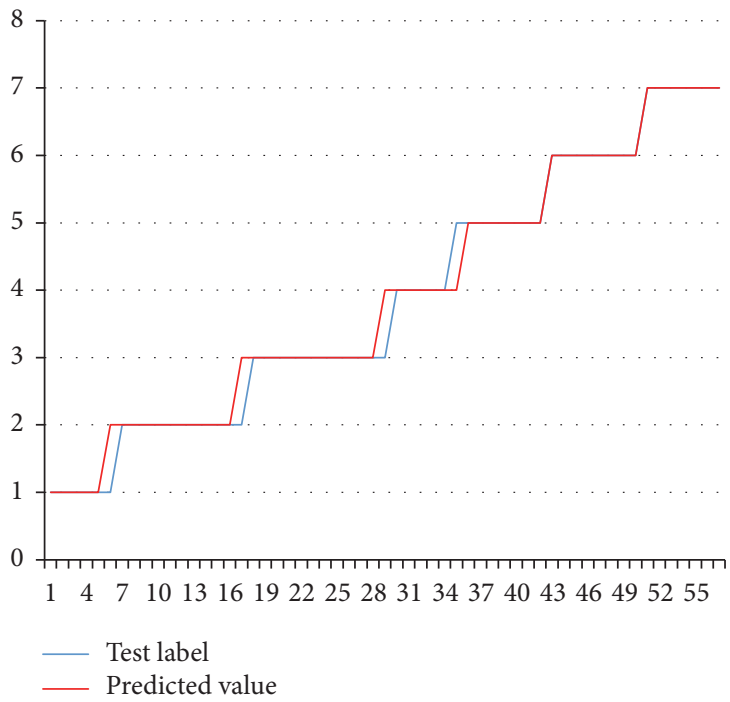

(c)

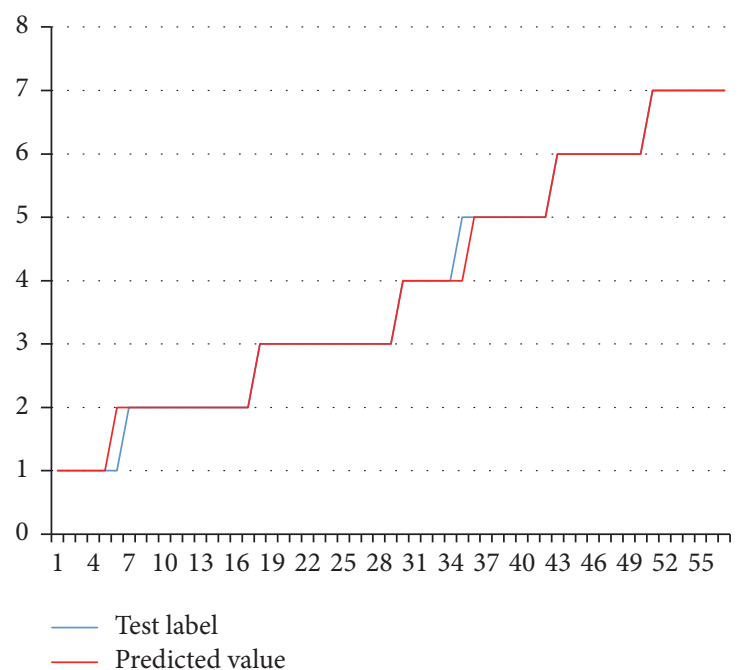

(b)

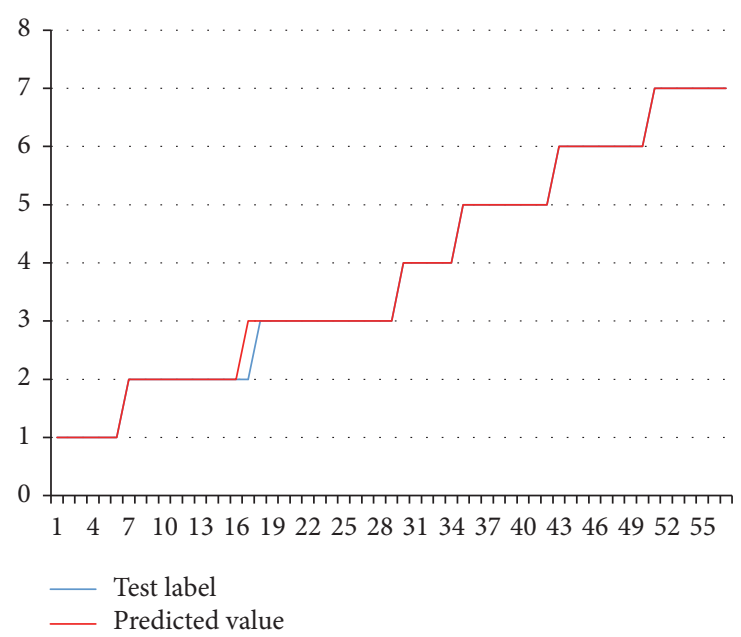

(d)

FIGURE 18: The gait phase recognition results of four cycles acquired from four different subjects. $K N N$, gait phase recognition model.

\section{Conflicts of Interest}

The authors declare that they have no conflicts of interest.

\section{Acknowledgments}

The work described in this paper is partially supported by the National Basic Research Program of China, 973 Program (2015CB351706), NSFC-Shenzhen Joint Fund for Robotics Research Center (U1613219), Guangdong Natural Science Foundation (2016A030313177), Shenzhen Fundamental Research and Discipline Layout project (JCYJ20150925163244742), and Shenzhen Fundamental Research (JCYJ20140901003938996). Finally, the authors thank the members of the SIAT exoskeleton team for supporting the research.

\section{References}

[1] Q. Jiang, S. Yang, and J. J. Sánchez-Barricarte, “Can China afford rapid aging?" SpringerPlus, vol. 5, no. 1, article 1107, 2016.

[2] N. Jabeur, N. Sahli, and S. Zeadally, "Enabling cyber physical systems with wireless sensor networking technologies, multiagent system paradigm, and natural ecosystems," Mobile Information Systems, vol. 2015, Article ID 908315, 2015.

[3] R. Ishak, S. Salleh, S. Olariu, and M. Abdul Aziz, "SPLAI: computational finite element model for sensor networks," Mobile Information Systems, vol. 2, no. 1, pp. 77-92, 2006.

[4] S. Kumar and S.-J. Park, "Probability model for data redundancy detection in sensor networks," Mobile Information Systems, vol. 5, no. 2, pp. 195-204, 2009.

[5] X. Hu, T. H. S. Chu, H. C. B. Chan, and V. C. M. Leung, "Vita: a crowdsensing-oriented mobile cyber-physical system," IEEE Transactions on Emerging Topics in Computing, vol. 1, no. 1, pp. 148-165, 2013. 
[6] X. Hu, J. Zhao, B.-C. Seet, V. C. M. Leung, T. H. S. Chu, and H. Chan, "S-aframe: agent-based multilayer framework with context-aware semantic service for vehicular social networks," IEEE Transactions on Emerging Topics in Computing, vol. 3, no. 1, pp. 44-63, 2015.

[7] T. Lenzi, S. M. M. De Rossi, N. Vitiello, and M. C. Carrozza, "Intention-based EMG control for powered exoskeletons," IEEE Transactions on Biomedical Engineering, vol. 59, no. 8, pp. 21802190, 2012.

[8] Y. Sankai, "HAL: Hybrid assistive limb based on cybernics," Springer Tracts in Advanced Robotics, vol. 66, pp. 25-34, 2010.

[9] K. Suzuki, G. Mito, H. Kawamoto, Y. Hasegawa, and Y. Sankai, "Intention-based walking support for paraplegia patients with robot suit HAL," Advanced Robotics, vol. 21, no. 12, pp. 14411469, 2007.

[10] A. Pantall and D. Ewins, "Muscle activity during stance phase of walking: comparison of males with transfemoral amputation with osseointegrated fixations to nondisabled male volunteers," Journal of Rehabilitation Research and Development, vol. 50, no. 4, pp. 499-514, 2013.

[11] S. Murray, K. H. Ha, C. Hartigan, and M. Goldfarb, "An assistive control approach for a lower-limb exoskeleton to facilitate recovery of walking following stroke," IEEE Transactions on Neural Systems \& Rehabilitation Engineering A Publication of the IEEE Engineering in Medicine \& Biology Society, vol. 23, no. 3, pp. 441-449, 2015.

[12] H. Kazerooni, R. Steger, and L. Huang, "Hybrid control of the berkeley lower extremity exoskeleton (BLEEX)," International Journal of Robotics Research, vol. 25, no. 5-6, pp. 561-573, 2006.

[13] D. Liu, X. Wu, W. Du, C. Wang, and T. Xu, "Gait phase recognition for lower-limb exoskeleton with only joint angular sensors," Sensors, vol. 16, no. 10, p. 1579, 2016.

[14] S. Oh, E. Baek, S.-K. Song, S. Mohammed, D. Jeon, and K. Kong, "A generalized control framework of assistive controllers and its application to lower limb exoskeletons," Robotics and Autonomous Systems, vol. 73, pp. 68-77, 2015.

[15] S. M. H. J. Jaegers, L. D. W. Vos, P. Rispens, and A. L. Hof, "The relationship between comfortable and most metabolically efficient walking speed in persons with unilateral above-knee amputation," Archives of Physical Medicine and Rehabilitation, vol. 74, no. 5, p. 521, 1993.

[16] D. Winter, "Human balance and posture control during standing and walking," Gait and Posture, vol. 3, no. 4, pp. 193-214, 1995.

[17] GB10000, "Human dimensions of Chinese adults," in General Administration of Quality Supervision, Inspection and Quarantine of the Peoples Republic of China, 1988.

[18] G. Q. Duan, "Manufacturing technique of aluminium-clad steel wire and its application in overhead transmission conductor," Electric Wire \& Cable, 2005.

[19] F. Yamasaki, K. Hosoda, and M. Asada, "An energy consumption based control for humanoid walking," in proceeding of the IEEE/RSJ International Conference on Intelligent Robots and Systems IEEE Xplore, vol. 3, pp. 2473-2477, 2002.

[20] N. Sánchez, A. M. Acosta, A. H. A. Stienen, and J. P. A. Dewald, "A multiple degree of freedom lower extremity isometric device to simultaneously quantify hip, knee, and ankle torques," IEEE Transactions on Neural Systems and Rehabilitation Engineering, vol. 23, no. 5, pp. 765-775, 2015.

[21] C. Li, S. T. Hsieh, and D. I. Goldman, "Multi-functional foot use during running in the zebra-tailed lizard (Callisaurus draconoides)," The Journal of Experimental Biology, vol. 215, no. 18, pp. 3293-3308, 2012.

[22] H. Kagaya, S. Ito, T. Iwami, G. Obinata, and Y. Shimada, "A computer simulation of human walking in persons with joint contractures," Tohoku Journal of Experimental Medicine, vol. 200, no. 1, pp. 31-37, 2003.

[23] K. C. Foucher, B. R. Schlink, N. Shakoor, and M. A. Wimmer, "Sagittal plane hip motion reversals during walking are associated with disease severity and poorer function in subjects with hip osteoarthritis," Journal of Biomechanics, vol. 45, no. 8, pp. 1360-1365, 2012.

[24] J. E. Zachazewski, P. O. Riley, and D. E. Krebs, "Biomechanical analysis of body mass transfer during stair ascent and descent of healthy subjects," Journal of Rehabilitation Research and Development, vol. 30, no. 4, pp. 412-422, 1993.

[25] L. A. Livingston, J. M. Stevenson, and S. J. Olney, "Stairclimbing kinematics on stairs of differing dimensions," Archives of Physical Medicine and Rehabilitation, vol. 72, no. 6, p. 398, 1991.

[26] S. L. Pavão, A. N. Santos, A. B. Oliveira, and N. A. C. F. Rocha, "Postural control during sit-to-stand movement and its relationship with upright position in children with hemiplegic spastic cerebral palsy and in typically developing children," Brazilian Journal of Physical Therapy, vol. 19, no. 1, pp. 18-25, 2015.

[27] G. Trevelyan, “Observational gait analysis," 238. rev.sfr.net.

[28] S. Nuzik, R. Lamb, A. VanSant, and S. Hirt, "Sit-to-stand movement pattern. A kinematic study," Physical Therapy, vol. 66, no. 11, pp. 1708-1713, 1986.

[29] M. J. Stephens and J. F. Yang, "Loading during the stance phase of walking in humans increases the extensor EMG amplitude but does not change the duration of the step cycle," Experimental Brain Research, vol. 124, no. 3, pp. 363-370, 1999. 

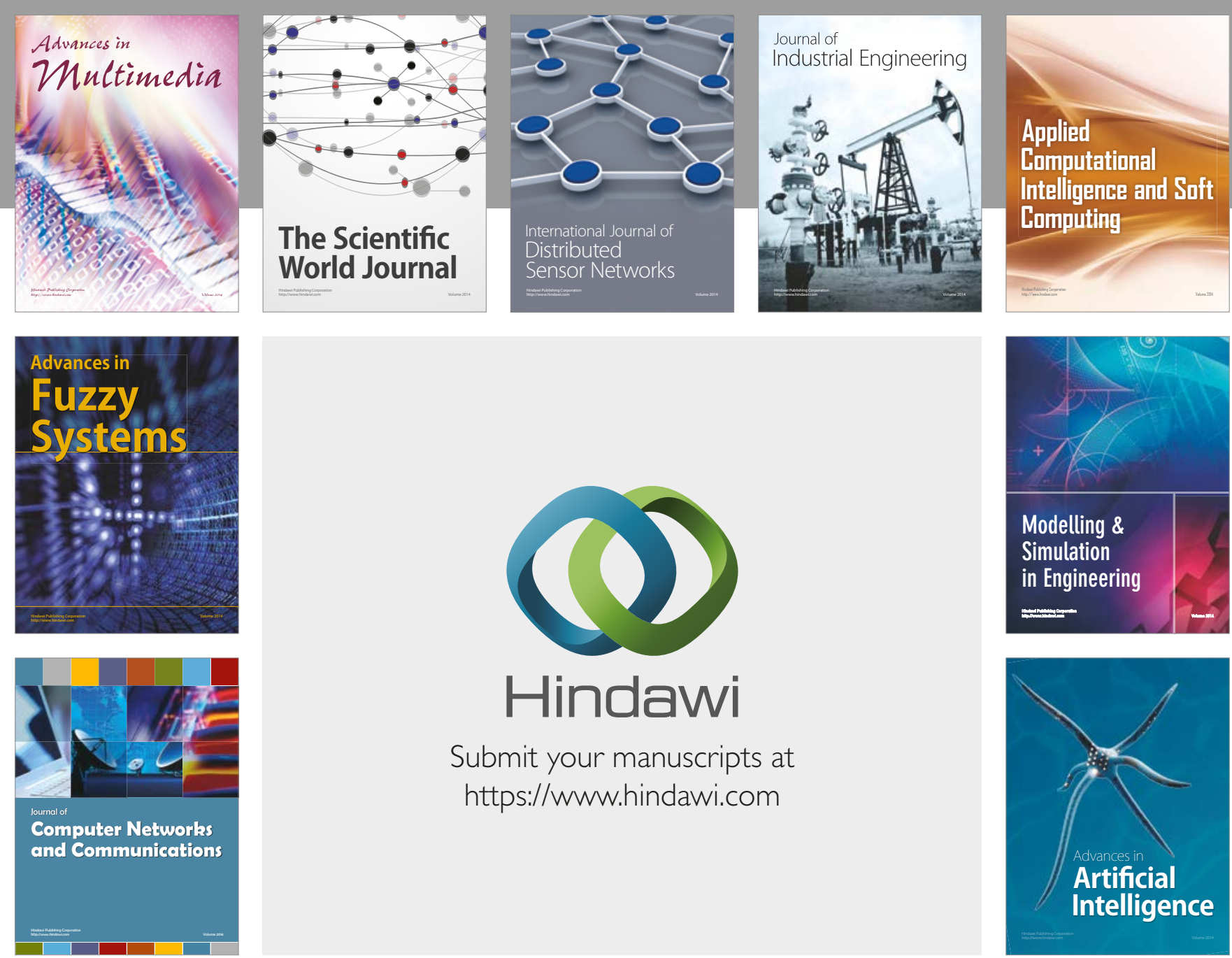

\section{Hindawi}

Submit your manuscripts at

https://www.hindawi.com
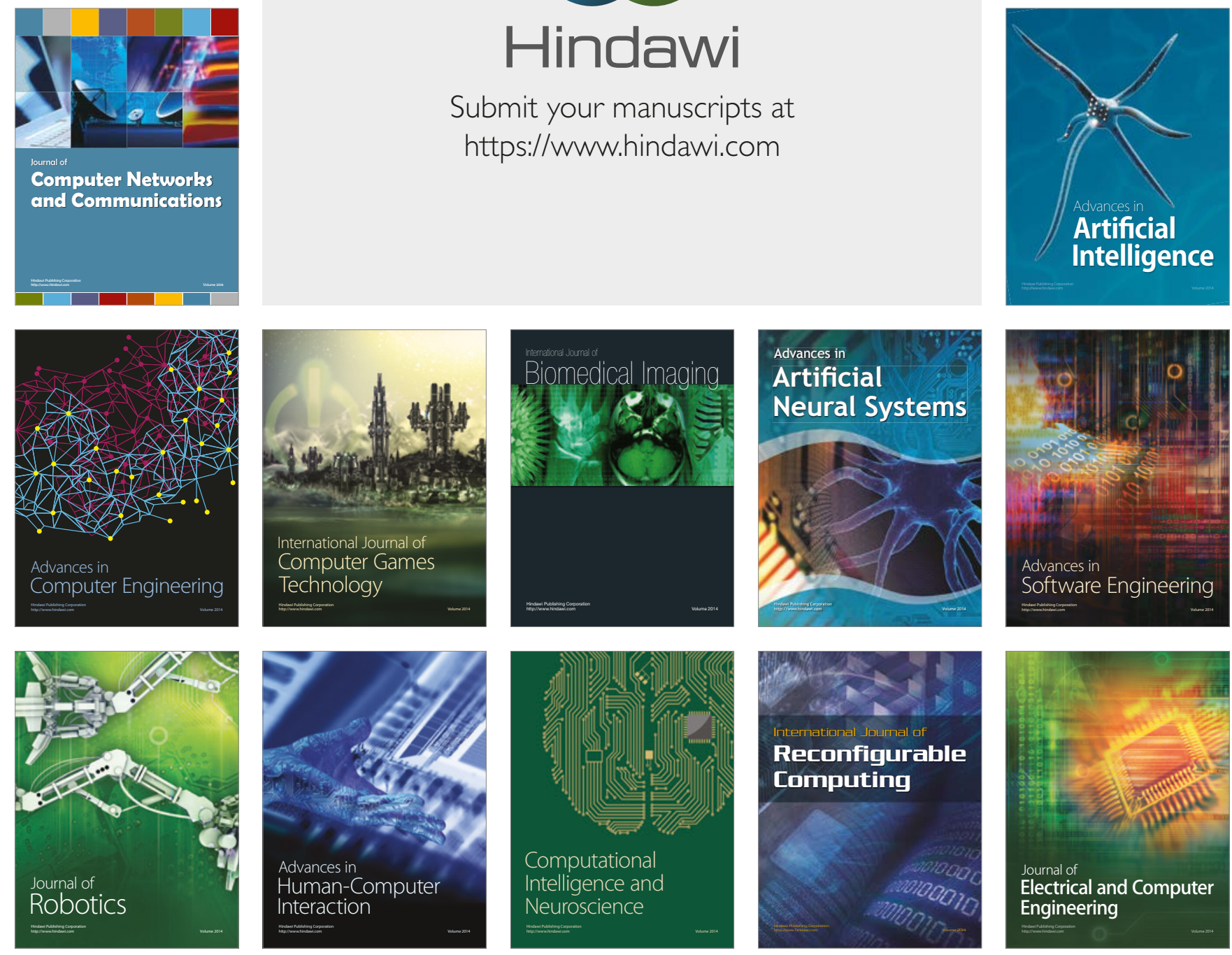